\title{
Rehabilitative ultrasound imaging for the assessment of selected morphometric parameters of the flexor hallucis longus muscle in healthy individuals: An inter- and intra- rater reliability study
}

\author{
Type \\ Research paper \\ Keywords \\ ultrasound, reliability, flexor hallucis longus, rater
}

\begin{abstract}
Introduction

Ultrasound imaging (USI) is useful to evaluate structures of the foot to guide treatment, but the reliability of USI technique needs to be clarified. The goal of the study was to evaluate the intra- and inter-examiner reliability of USI image capture, and measurement of the cross-sectional area (CSA) and thickness of the flexor hallucis longus (FHL) for experienced and novice examiners
\end{abstract}

Material and methods

FHL images were captured for 20 healthy adults. Reliability of image capture was evaluated between images repeated at 10-min interval for an experienced and a novice examiner. Reliability of imagebased measurements was evaluated for one experienced and one novice rater, using all images. The intra-class correlation coefficient (ICC) and the standard error of measurement (SEM) were calculated

Results

Intra-examiner reliability of image capture for the FHL muscle examined by USI was excellent for both thickness (ICC3,1, 0.944-0.976; SEM, 6.8\%-10.0\%) and CSA (ICC3,1, 0.954-0.979; SEM, $10.8 \%-16.5 \%$ ), with no effect of examiner experience. Reliability was also excellent for measurement of thickness (ICC3,1, 0.954-0.972; SEM, 1.2\%-9.6\%) and CSA (ICC3,1, 0.961-0.986; SEM, $9.2 \%-14.1 \%)$, with no effects of experience.

\section{Conclusions}

Reliability of image capture and image-based measurements developed by USI of CSA and thickness for the FHL muscle in healthy individuals was excellent, independent of the examiner experience. 
1 Rehabilitative ultrasound imaging for the assessment of selected morphometric parameters of the flexor hallucis longus muscle in healthy individuals: An inter- and intra- rater reliability study

Abstract: Background: Ultrasound imaging (USI) is useful to evaluate structures of the foot to guide treatment, but the reliability of USI technique needs to be clarified. The goal of the study was to evaluate the intra- and inter-examiner reliability of USI image capture, and measurement of the cross-sectional area (CSA) and thickness of the flexor hallucis longus (FHL) for experienced and novice examiners. Methods: FHL images were captured for 20 healthy adults. Reliability of image capture was evaluated between images repeated at 10-min interval for an experienced and a novice examiner. Reliability of image-based

11 measurements was evaluated for one experienced and one novice rater, using all images.

12 The intra-class correlation coefficient (ICC) and the standard error of measurement (SEM)

13 were calculated. Results: Intra-examiner reliability of image capture was excellent for both thickness ( $\mathrm{ICC}_{3,1}, 0.944-0.976$; SEM, 6.8\%-10.0\%) and CSA ( ICC $_{3,1}, 0.954-0.979$; SEM, $10.8 \%-16.5 \%$ ), with no effect of examiner experience. Reliability was also excellent for measurement of thickness ( $\mathrm{ICC}_{3,1}, 0.954-0.972$; SEM, 1.2\%-9.6\%) and CSA $\left(\mathrm{ICC}_{3,1}\right.$, 0.961-0.986; SEM, 9.2\%-14.1\%), with no effects of experience. Conclusion: Reliability of image capture and image-based measurements of CSA and thickness for the FHL muscle in healthy individuals was excellent, independent of the examiner experience. 


\section{Introduction}

The flexor hallucis longus is the primary plantar flexor of the first metatarsophalangeal (MTP) and interphalangeal (IP) joints of the great toe; it also restrains the passive dorsiflexion at the first MTP joint and contributes to ankle plantar flexion [1]. FHL tendinopathy is one of the most common pathological conditions of the ankle and foot among active people [2].

Ultrasound imaging (USI) is considered as a safe, non-invasive, and valid method to measure muscle morphology to inform diagnosis, and to compare the cross-sectional area (CSA) and thickness of the FHL in healthy subjects [3], subjects with pes planus [4,5] , and dancers [6]. Extending the application of USI in clinical practice would require assessing the reliability of measurements obtained.

Mickleet al. [7] evaluated the intra-examiner reliability of USI to measure the morphology of the primary toe flexor muscles, while Crofts et al. [8] analyzed the interexaminer reliability of USI for selected foot structures. Both of these studies suggest that USI offers the opportunity to quantify the structures of the foot for a better understanding of their functional contribution. Researchers have identified the need for determining the reliability of USI not only for measurement (USI-based calculation) but also for image capture (patient positioning), both of which might be influenced by the experience of the examiner. ValeraCalero et al. [9] have previously investigated the reliability of US image capture and measurement of the CSA of the cervical multifidus muscle as a function of the examiner's experience in asymptomatic individuals, with excellent reliability reported.

The objective of this study was to determine intra- and inter-examiner reliability of US image capture (probe assessment/subject positioning) and measurement (scan/image assessment) for the CSA and thickness of the FHL as a function of the examiner's experience. Understanding the architecture of the FHL has implications for invasive physiotherapeutic therapies, as most of them use ultrasound guidance to guarantee accuracy $[10,11]$.

\section{Materials and Methods}

\section{Participants}

Twenty healthy volunteers were recruited from private physiotherapy clinics between April and May 2020. The procedures were approved by the local Ethics Committee (1046$\mathrm{N}-19$ ) in accordance with the current national and international laws and regulations governing the use of human subjects. Before participating in the study, the subjectss were 
fully informed about the protocol and written informed consent was obtained from each participant before testing. Enrolled participants were females over the age of 18 years and with no history of ankle pain over the previous year. The exclusion criteria were as follows: acute lower limb injury within the previous six months; prior ankle surgery; history of low back pain; history of peripheral nerve impairment; congenital/acquired foot deformities; any other medical condition or treatment affecting muscle tone.

\section{Imaging capturing and FHL measurement protocol}

For ultrasound evaluation of the FHL muscle, the participants lay in the prone position, with the feet unsupported at the edge of the bed. The prone position is normally used for invasive treatment techniques $[10,11]$. Using a previously described procedure $[10,11]$, the FHL muscle was located at the midpoint of the distance between the fibular head and the inferior border of the lateral malleolus on the posterior aspect of the fibula, using the Logiq US machine (GE Healthcare, USA; Fig 1). Images were captured using a $12 \mathrm{MHz}$ linear transducer, in the longitudinal and transverse plane of the muscle. Image-based measures were performed using ImageJ (version 1.53a; Bethesda, MD, USA).

\section{[Figure 1]}

\section{Reliability protocol}

Reliability of US image capture was evaluated for one novice (1 year of practice) and one experienced (13 years of practice) examiner, both using the prone patient positioning as previously described. Each examiner captured two images of the FHL muscle, with a 10-min period between the two image-captures, with participant positioning required for each assessment. Reliability of image-based measurements was evaluated, again, for one experienced and a novice rater, with the same years of experience as the examiners previously described. Measurements were performed in blinded fashion (no identification if images were obtained by the novice or experienced examiner), with images coded using numerical codes.

[Figure 1]

\section{Reliability of image-based measurements}

The intra-rater reliability of image-based measurements of the CSA and thickness of the FHL was evaluated for two rater (experienced/novice), and using images obtained from the 
experienced and novice operators. Inter-rater reliability was evaluated for measures of CSA and thickness using the images obtained during the first imaging assessment from both the experienced and novice examiner. We also evaluated inter-rater reliability of measurement of the FHL CSA and thickness for the experienced and novice examiners for all images obtained at once. For intra-examiner reliability, each examiner repeated measurements of CSA and thickness at a 1-week interval.

\section{Statistical analysis}

Statistical analysis was performed using SPSS (v.22, IBM; Armonk, NY: IBM Corp.), with an $\alpha$ error of 0.05 and a $\beta$ error of 0.2 for both intra- and inter-examiner/rater reliability. The Shapiro-Wilk test was employed for the normality assumption. A 2-way, mixed-model, consistency-type, intra-class correlation coefficients (ICC) was calculated to test the intraand inter-rater reliability. Reliability was defined as fair (ICC<0.50), moderate $(0.50<\mathrm{ICC}<0.75)$, good $(0.75<\mathrm{ICC}<0.90)$, or excellent (ICC >0.90) [12]. In addition, the ICC and associated standard deviation (SD) were used to calculate the standard error measurement as a measure of the precision of measurement, as follows: SEM $(\%)=(\operatorname{SDx} \sqrt{ } 1-\mathrm{ICC}) \times 100$.

\section{Results}

The age and relevant anthropometric data for the study group are summarized in Table 1.

\section{[Table 1]}

Reliability results for image capture and FHL thickness is reported in Table 2. The results showed in this table demonstrate that reliability was excellent, overall, with ICC values ranging between 0.944 to 0.976 , with SEM values of $6.8 \%$ to $10.0 \%$. There was no difference between the experienced and novice examiner, with inter-examiner ICCs ranging between 0.983 and 0.994 , with SEM values of $0.1 \%$ to $5.3 \%$.

\section{[Table 2]}

Reliability of image-based measurement of FHL thickness is reported in Table 3. The intra-rater reliability was excellent, with ICCs ranging between 0.954 and 0.972 and SEM values of $1.2 \%$ to $9.6 \%$. There was no difference in reliability between the experienced and novice rater, with inter-rater ICCs of 0.950 to 0.980 and SEM values of $6.2 \%$ to $8.5 \%$. 
Reliability of image capture for CSA is reported in Table 4. The reliability was again excellent, with ICCs of 0.954 to 0.979 and SEM values of $10.8 \%$ to $16.5 \%$. There was no

124 difference between the experienced and novice examiner, with inter-rater ICC values of 0.986 125 to 0.984 and SEM values of $8.9 \%$ to $15.0 \%$. reliability was excellent, with ICCs of 0.961 to 0.986 and SEM values of $9.2 \%$ to $14.1 \%$. Again, there was no different between the experienced and novice rater, with inter-rater ICCs of 0.961 to 0.991 and SEM values of $6.9 \%$ to $15.1 \%$.

[Table 5]

\section{Discussion}

Our findings indicate high reliability of image capture of the CSA and thickness of the

134 FHL, as well as for image-based measurements of CSA and thickness This reliability would

135 be independent of the examiner experience. To the authors' knowledge, this is the first study

136 that has evaluated the reliability of both USI capture (positioning) and measurement for the

137 FHL muscle considering the experience of the examiner. Our findings are consistent with 138 previous studies which have reported ICC values $>0.90[7,8]$, and underscore the feasibility 139 of using USI. Despite the high prevalence of FHL tendinopathy, there is limited evidence of 140 the role of the FHL in foot mechanics, and of the impact of an injury in this muscle [13].

141 Injuries to the FHL muscle and its tendon are often overlooked. In specific populations, 142 however, appropriate assessment of this muscle is essential, such as in dancers who rely on 143 the FHL for dynamic stability of the foot during movements that require alternation between 144 extreme plantar flexion and dorsiflexion [14]. Dancers are high-performance athletes who 145 are particularly susceptible to lower extremities -specially foot- injuries [15-19]. By 146 demonstrating the reliability of USI of the FHL, this study may offer a novel approach to 147 assess and quantify the injury status of the FHL, as well as providing the image guidance 148 needed for invasive therapies (such as needle insertion) targeting this muscle. The impact of 149 any injury in professional athletes justifies the requirement of improving assessment and 150 treatment techniques and guarantee their reliability [20,21]. Crofts et al. [7] and Mickle et al. 151 [8] placed individuals in a supine position to locate the FHL. However, other studies [6,22], 
and in particular those targeting invasive therapies such as US-guided percutaneous neuromodulation, have used a prone position for patient placement, with feet unsupported at the end of the plinth. In the present study, prone position was selected, as it has been reported to be more comfortable for the patient, provided greater lower limb stability, and facilitated handling of the probe with one hand and the possible needle with the other $[9,10]$. Due to these facts, assessing USI reliability in this position was considered to have more implications for potential use in the field of invasive techniques.

The authors acknowledge the limitations of this study. Foremost, the small sample size of the study group. The characteristics of the sample, with median age of 21 years, healthy BMI, no previous history of FHL injury and no lower limb impairments, might be not representative of the range of patients with FHL tendinopathy. However this results may be useful in future studies in rehabilitation medicine in other populations. Future lines of research might include repeated assessment of reliability in patients with tendinopathy, as well as in athletes with suspected impairments in FHL function. Further research with a higher sample size is needed.

\section{Conclusions}

USI examination of the FHL muscle is highly reliable for the evaluation of the CSA and thickness of the muscle in healthy participants, not depending on the experience of the examiner. Continued assessment of the reliability of US image capture and image-based measurements of FHL morphometric parameters would be important to extend the importance of USI in clinical practice. 


\section{References}

176 1. Michelson J, Dunn L. Tenosynovitis of the flexor hallucis longus: A clinical study of 177 the spectrum of presentation and treatment. Foot Ankle Int 2005;26(4):291-303.

2. Khan K, Brown J, Way S, Vass N, Crichton K, Alexander R, et al. Overuse Injuries in Classical Ballet. Vol. 19, Sports Medicine 1995. p. 341-57.

3. Latey PJ, Burns J, Nightingale EJ, Clarke JL, Hiller CE. Reliability and correlates of cross-sectional area of abductor hallucis and the medial belly of the flexor hallucis brevis measured by ultrasound. J Foot Ankle Res 2018;11(1).

4. Angin S, Crofts G, Mickle KJ, Nester CJ. Ultrasound evaluation of foot muscles and

5. Angin S, Mickle KJ, Nester CJ. Contributions of foot muscles and plantar fascia

6. De-la-Cruz-Torres B, Barrera-García-Martín I, De la Cueva-Reguera M, BravoAguilar M, Blanco-Morales M, Navarro-Flores E, et al. Does Function Determine the Structure? Changes in Flexor Hallucis Longus Muscle and the Associated Performance Related to Dance Modality: A Cross-Sectional Study. Medicina (Kaunas) 2020;56(4).

7. Mickle KJ, Nester CJ, Crofts G, Steele JR. Reliability of ultrasound to measure morphology of the toe flexor muscles J Foot Ankle Res. 2013;6(1).

8. Crofts G, Angin S, Mickle KJ, Hill S, Nester CJ. Reliability of ultrasound for measurement of selected foot structures Gait Posture. 2014;39(1):35-9.

9. Valera-Calero JA, Sánchez-Jorge S, Álvarez-González J, Ortega-Santiago R, Cleland JA, Fernández-de-Las-Peñas $\mathrm{C}$, et al. Intra-rater and inter-rater reliability of rehabilitative ultrasound imaging of cervical multifidus muscle in healthy people: Imaging capturing and imaging calculation. Musculoskelet Sci Pract 2020;48:102158.

10. de-la-Cruz-Torres B, Barrera-García-Martín I, Romero-Morales C. Comparative Effects of One-Shot Electrical Stimulation on Performance of the Flexor Hallucis Longus Muscle in Professional Dancers: Percutaneous Versus Transcutaneous? Neuromodulation. 2019;

11. de la Cruz-Torres B, Barrera-García-Martín I, Albornoz-Cabello M. Immediate effects of ultrasound-guided percutaneous neuromodulation versus physical exercise on randomised clinical trial Acupunct Med. 2019;37(2):91-7. 
12. Koo TK, Li MY. A Guideline of Selecting and Reporting Intraclass Correlation Coefficients for Reliability Research. J Chiropr Med 2016;15(2):155-63.

13. Vosseller JT, Dennis ER, Bronner S. Ankle Injuries in Dancers. J Am Acad Orthop Surg 2019;27(16):582-9.

14. Hamilton WG. Foot and ankle injuries in dancers. Clin Sports Med 1988;7(1):14373.

15. De-la-Cruz-Torres B, Barrera-García-Martín I, Valera-Garrido F, Minaya-Muñoz F, Romero-Morales C. Ultrasound-Guided Percutaneous Needle Electrolysis in Dancers with Chronic Soleus Injury: A Randomized Clinical Trial. Evid Based Complement Alternat Med 2020;2020:4156258.

16. Bronner S, Novella T, Becica L. Management of a delayed-union sesamoid fracture in a dancer. J Orthop Sports Phys Ther 2007;37(9):529-40.

17. Bronner S, Ojofeitimi S, Rose D. Repair and rehabilitation of extensor hallucis longus and brevis tendon lacerations in a professional dancer. J Orthop Sports Phys Ther 2008;38(6):362-70.

18. Filipa A, Barton K. Physical Therapy Rehabilitation of an Adolescent Preprofessional Dancer Following Os Trigonum Excision: A Case Report. J Orthop Sport Phys Ther 2017;48(3):194-203.

19. Ojofeitimi S, Bronner S, Becica L. Conservative Management of Second Metatarsophalangeal Joint Instability in a Professional Dancer: A Case Report. J Orthop Sport Phys Ther 2016;46(2):114-23.

20. Hamilton WG. Posterior ankle pain in dancers. Clin Sports Med 2008;27(2):263-77.

21. Luk P, Thordarson D, Charlton T. Evaluation and management of posterior ankle pain in dancers. J Danc Med Sci Off Publ Int Assoc Danc Med Sci 2013;17(2):79-83.

22. de-la-Cruz-Torres B, Barrera-García-Martín I, Cueva-Reguera M de la, Bravo-Aguilar M, Abuin-Porras V, Romero-Morales C. Ultrasound imaging features of the Achilles tendon in dancers. Is there a correlation between the imaging and clinical findings? A cross-sectional study. Phys Ther Sport Off J Assoc Chart Physiother Sport Med 2020;43:181-7. 
Table 1. Sociodemographic data

\begin{tabular}{lc} 
Data & Total $(\mathbf{n}=\mathbf{2 0})$ \\
\hline Age (years) & $21.05 \pm 2.3$ \\
Weight $(\mathrm{kg})$ & $56.30 \pm 5.6$ \\
Height $(\mathrm{m})$ & $1.65 \pm 0.0$ \\
BMI $\left(\mathrm{kg} / \mathrm{m}^{2}\right)$ & $20.46 \pm 0.6$ \\
\hline
\end{tabular}

Abbreviations: body mass index, BMI 
Table 2. Intra- and inter-rater reliability of imaging for the FHL thickness capturing

\section{ICC $(95 \% \mathrm{CI})$}

SD

SEM

FHL Thickness (cm)

Intra- Rater (A)

Experienced Rater

$0.957(0.892-0.983)$

0.435

$6.8 \%$

Novice Rater

$0.969(0.922-0.988)$

0.432

$7.6 \%$

Intra- Rater (B)

Experienced Rater

$0.963(0.909-0.985)$

0.426

$8.1 \%$

Novice Rater

$0.976(0.938-0.990)$

0.426

$6.5 \%$

Intra-Rater (C)

Experienced Rater

$0.964(0.909-0.986)$ *

0.463

$8.7 \%$

Novice Rater

$0.969(0.922-0.988)$

0.450

$7.9 \%$

Intra- Rater (D)

Experienced Rater

$0.978(0.945-0,991)$

0.450

$6.6 \%$

Novice Rater

$0.944(0.856-0.978)$

0.426

$10.0 \%$

Inter- Rater (A)

$0.991(0.976-0.996)$

0.418

$0.1 \%$

Inter- Rater (B)

$0.983(0.957-0.993)$

0.412

$5.3 \%$

Inter- Rater (C)

0.994 (0.986- 0.998)

0.452

$0.1 \%$

Inter- Rater (D)

$0.991(0.977-0.996)$

0.435

$0.1 \%$ 
Table 3. Intra- and inter-rater reliability of imaging for the FHL thickness calculation

\section{ICC $(95 \%$ CI $)$}

SD

SEM

FHL thickness (cm)

Intra- Rater (A)

Experienced Rater

$0.968(0.920-0.987)$

0.455

$8.1 \%$

Novice Rater

$0.969(0.922-0.988)$

0.432

$7.6 \%$

Intra- Rater (B)

Experienced Rater

0.963 (0.909- 0.985)

0.432

$8.3 \%$

Novice Rater

$0.972(0.930-0.989)$

0.442

$7.3 \%$

Intra- Rater (C)

Experienced Rater

$0.954(0.885-0.982)$

0.450

$9.6 \%$

Novice Rater

$0.966(0.913-0.986)$

0.443

$8.1 \%$

Intra- Rater (D)

Experienced Rater

$0.973(0.931-0.989)$

0.454

$1.2 \%$

Novice Rater

$0.968(0.920-0.987)$

0.431

$7.7 \%$

Inter- Rater (A)

$0.958(0.897-0.983)$

0.430

$7.8 \%$

Inter- Rater (B)

$0.967(0.915-0.987)$

0.415

$7.5 \%$

Inter- Rater (C)

$0.961(0.902-0.985)$

0.435

$8.5 \%$

Inter- Rater (D)

$0.980(0.950-0.992)$

0.444

$6.2 \%$ 
Table 4. Intra- and inter-rater reliability of imaging for the FHL CSA capturing

\section{ICC $(95 \%$ CI $)$}

SD

SEM

FHL CSA $\left(\mathrm{cm}^{2}\right)$

Intra- Rater (A)

Experienced Rater

$0.967(0.913-0.987)$

0.733

$13.3 \%$

Novice Rater

$0.971(0.928-0.988)$

0.736

$12.5 \%$

Intra- Rater (B)

Experienced Rater

$0.979(0.948-0.992)$

0.749

$10.8 \%$

Novice Rater

$0.962(0.904-0.985)$

0.738

$14.3 \%$

Intra-Rater $(C)$

Experienced Rater

0.967 (0.917- 0.987)

0.694

$12.6 \%$

Novice Rater

$0.954(0.882-0.982)$

0.700

$16.5 \%$

Intra- Rater (D)

Experienced Rater

$0.970(0.925-0.988)$

0.744

$12.8 \%$

Novice Rater

$0.962(0.907-0.985)$

0.772

$15.0 \%$

Inter- Rater (A)

$0.984(0.953-0.994)$

0.710

$8.9 \%$

Inter- Rater (B)

$0.964(0.909-0.986)$

0.760

$14.4 \%$

Inter- Rater (C)

$0.984(0.960-0.994)$

0.737

$9.3 \%$

Inter- Rater (D)

$0.965(0.913-0.986)$

0.732

$13.6 \%$

Abbreviations: SEM, standard error measurement; SD, standard deviation 
Table 5. Intra- and inter-rater reliability of imaging for the FHL CSA calculation.

\section{ICC $(95 \%$ CI $)$}

SD

SEM

FHL CSA $\left(\mathrm{cm}^{2}\right)$

Intra- Rater (A)

Experienced Rater

$0.964(0.910-0.986)$

0.727

$13.7 \%$

Novice Rater

$0.964(0.909-0.986)$

0.723

$13.7 \%$

Intra- Rater (B)

Experienced Rater

0.979 (0.947- 0.991)

0.748

$10.8 \%$

Novice Rater

$0.972(0.930-0.989)$

0.757

$12.6 \%$

Intra-Rater $(C)$

Experienced Rater

0.986 (0.965- 0.994)

0.781

$9.2 \%$

Novice Rater

0.961 (0.898- 0.985)

0.714

$14.1 \%$

Intra- Rater (D)

Experienced Rater

$0.974(0.932-0.990)$

0.734

$11.8 \%$

Novice Rater

$0.966(0.915-0.986)$

0.769

$14.1 \%$

Inter- Rater (A)

0.967 (0.910- 0.988)

0.713

$12.9 \%$

Inter- Rater (B)

$0.967(0.918-0.987)$

0.715

$12.9 \%$

Inter- Rater (C)

$0.991(0.977-0.996)$

0.735

$6.9 \%$

Inter- Rater (D)

$0.961(0.902-0.985)$

0.765

$15.1 \%$ 


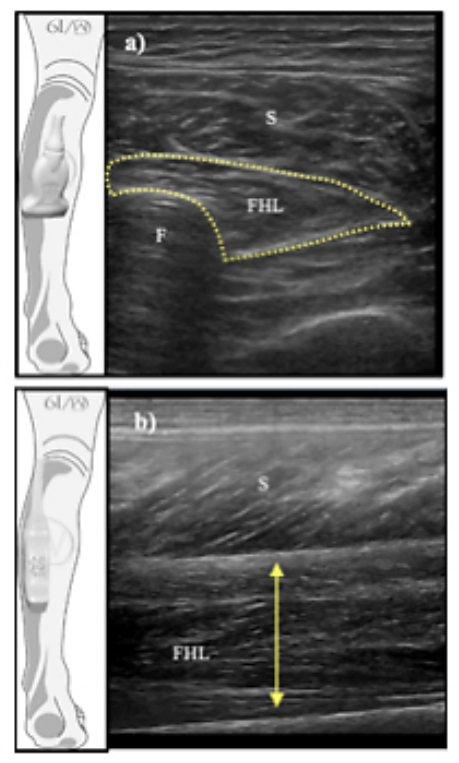

Ultrasound imaging of the flexor hallucis longus thickness and CSA 